A soft $x$-ray transmission grating imaging-spectrometer for the National Ignition Facility

A. S. Moore, T. M. Guymer, J. L. Kline, J. Morton, M. Taccetti, N. E. Lanier, C. Bentley, J. Workman, B. Peterson, K. Mussack, J. Cowan, R. Prasad, M. Richardson, S. Burns, D. H. Kalantar, L. R. Benedetti, P. Bell, D. Bradley, W. Hsing, M. Stevenson

May 8, 2012

19th Topical Conference on High-Temperature Plasma Diagnostics Monterey, CA, United States

May 6, 2012 through May 10, 2012 
This document was prepared as an account of work sponsored by an agency of the United States government. Neither the United States government nor Lawrence Livermore National Security, LLC, nor any of their employees makes any warranty, expressed or implied, or assumes any legal liability or responsibility for the accuracy, completeness, or usefulness of any information, apparatus, product, or process disclosed, or represents that its use would not infringe privately owned rights. Reference herein to any specific commercial product, process, or service by trade name, trademark, manufacturer, or otherwise does not necessarily constitute or imply its endorsement, recommendation, or favoring by the United States government or Lawrence Livermore National Security, LLC. The views and opinions of authors expressed herein do not necessarily state or reflect those of the United States government or Lawrence Livermore National Security, LLC, and shall not be used for advertising or product endorsement purposes. 


\title{
A soft $x$-ray transmission grating imaging-spectrometer for the National Ignition Facility
}

\author{
A. S. Moore ${ }^{1}$, T. M. Guymer ${ }^{1}$, J. L. Kline ${ }^{2}$, J. Morton ${ }^{1}$, M. Taccetti ${ }^{2}$, N. E. Lanier ${ }^{2}$, C. Bentley ${ }^{1}$, \\ J. Workman ${ }^{2}$, B. Peterson ${ }^{2}$, K. Mussack ${ }^{2}$, J. Cowan', R. Prasad ${ }^{3}$, M. Richardson ${ }^{3}$, S. Burns ${ }^{3}$, \\ D. H. Kalantar ${ }^{3}$, L. R. Benedetti ${ }^{3}$, P. Bell ${ }^{3}$, D. Bradley ${ }^{3}$, W. Hsing $^{3}$, M. Stevenson ${ }^{1}$
}

1 Directorate Science and Technology, AWE Aldermaston, Reading, RG7 4PR, United Kingdom

2 Los Alamos National Laboratory, Los Alamos, NM 87545 USA

3 Lawrence Livermore National Laboratory, P.O. Box 808, Livermore, CA 94551-0808 USA

(Received XXXXX; accepted XXXXX; published online XXXXX)

(Dates appearing here are provided by the Editorial Office)

\begin{abstract}
A soft x-ray transmission grating spectrometer has been designed for use on high energy-density physics experiments at the National Ignition Facility (NIF); coupled to one of the NIF gated $x$-ray detectors (GXD) it records sixteen time-gated spectra between 250 and $1000 \mathrm{eV}$ with $100 \mathrm{ps}$ temporal resolution. The trade-off between spectral and spatial resolution leads to an optimized design for measurement of emission around the peak of a $100-300 \mathrm{eV}$ blackbody spectrum. Performance qualification results from the NIF, the Trident Laser Facility and VUV beamline at the National Synchrotron Light Source (NSLS), evidence a $<100 \mu \mathrm{m}$ spatial resolution in combination with a source-size limited spectral resolution that is $<10 \mathrm{eV}$ at photon energies of $300 \mathrm{eV}$.
\end{abstract}

\section{INTRODUCTION}

Understanding the behaviour of both dynamic laboratory astrophysics experiments and laser-fusion experiments (Moses 2009) (Back 2000) requires accurate measurement of the radiation field and material temperature and densities. Spectroscopy has often been used in such experiments to observe the turn-on of hard $x$-ray $(>1 \mathrm{keV})$ spectral lines characteristic to a tracer-layer embedded into the target (Hoarty 1999). Interpreting these results requires a number of assumptions, including the shape of the electron-energy distribution function that heats the tracer material. An alternative approach is to directly measure the emitted radiation field from the material that is heated. The low-energy $(<1 \mathrm{keV})$ spectrum associated with thermal emission from plasmas with $T_{r} 200 \mathrm{eV}$ is important since for a wide variety of experiments involving optically-thick, diffusive radiation this part of the spectrum dictates the behaviour of the radiation transport and resulting hydrodynamics. In particular, experiments studying diffusive radiation flow are sensitive to the shape of the spectrum since although they are by design close to local thermodynamic equilibrium (LTE) at all times, this does not mean that the radiation spectrum has to be Planckian. Rather, the detailed shape of the Planckian region, together with atomic physics simulations, provides a rich insight into the complex interplay between the plasma and radiation field, not least because it contains the majority of the energy in the problem and so can be very constraining for radiation hydrodynamics simulations.

\section{SPECTROMETER DESIGN}

Transmission grating spectrometers have been used to diagnose laser-produced plasmas in the past (Ceglio
1983) and have a number of advantages over reflective grating spectrometers, not least of which is implementation. In it's simplest form a direct line of sight spectrometer can be created by simple bounding of the grating with a pinhole-aperture. The diffraction efficiency is typically higher than for reflective gratings, and in the non-dispersive direction, there are no stringent requirements on the 'flatness' of a reflective grating optic. Finally, the direct line-of-sight or 'zero-order' is usually more easily blocked from a transmission grating than is sometimes possible from a reflective diffraction element. The primary disadvantage is typically the fragility of the transmission grating.

The typical plasma radiation temperatures achievable in the laboratory primarily determines the spectral range of interest, and for any diffractive element the lowest, and consequently most diffracted, energy is usually most constraining. High energy-density plasma physics experiments typically fall in the range of $\mathrm{T}_{\mathrm{r}}=100$ $350 \mathrm{eV}$, therefore to measure $\mathrm{x}$-radiation emission from the Planckian peak requires that the low-energy bound be 250$300 \mathrm{eV}$. To make optimal use of each grating, the positive and negative order diffraction is utilised, and therefore the high-energy bound is set only by the physical extent of the zero-order block necessary to prevent direct-line-of-sight debris from impacting the detector. The spectral and spatial resolution are determined by the specific use of the diagnostic being primarily related to the source-size and solid collection angle of the diagnostic. Finally, the detector used in conjunction with the spectrometer must be given careful consideration depending on the application. The spectrometer we describe here is typically coupled to a GXD (Ze 1992), although application with a streak camera is readily possible. Critical to this discussion is knowledge 


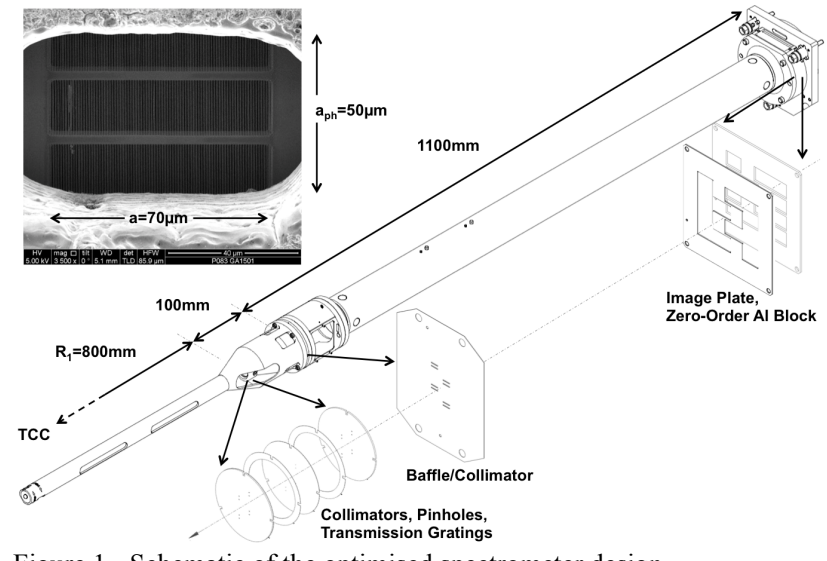

Figure 1 - Schematic of the optimised spectrometer design

of the wavelength sensitivity of either the streak camera photo-cathode and/or multi-channel plate (MCP) detector in a GXD, and characterising the spectral response of such detectors has been an area of considerable research over past decades (Rochau 2006). To correctly interpret the spectral data from a transmission grating diffraction pattern for the soft x-ray application described here, the most critical information is the relative change in sensitivity at the material edges of Carbon $(283 \mathrm{eV})$ and Oxygen $(543 \mathrm{eV})$, since this is of importance to reconstructing the spectrum. If information about the relative brightness of spectral features on either side of these edges is necessary, then this difficult measurement is required, however, if the spectral region of interest is limited to between 283 and $543 \mathrm{eV}$, then the smooth change in the detector response is relatively well-known. Importantly, since it is only the relative shape of the spectrum that is important for a measurement of the spectral shape, the absolute sensitivity is not required.

Given the requirements discussed above it is possible to begin to limit the design space available. For the GXD's described above, the MCP area is split into 4-strips that can be independently timed; each strip is $37.3 \times 7.5 \mathrm{~mm}$ and spaced $9.7 \mathrm{~mm}$ apart. Dispersing the spectrum along the long direction of each strip maximises the continuous spectral record, which together with the minimum energy requirement of $250 \mathrm{eV}$, tolerances in diagnostic pointing, and grating period $d=0.5 \mu \mathrm{m}$, sets the grating-to-detector distance, $R_{2}=1200 \mathrm{~mm}$ simply by the grating equation:

$$
n \lambda=d \sin \theta \quad \ldots(1)
$$

The grating aperture is limited to $50 \times 70 \mu \mathrm{m}$ $(a=70 \mu \mathrm{m})$ by a pinhole placed immediately in front of the grating as shown in the scanning electron microscope image inset to Figure 1. This produces a simple pinholecamera spatially imaging the $\mathrm{x}$-ray emission in the short direction of the MCP strip.

The target-to-grating distance $\left(R_{l}\right)$ determines the spectral and spatial resolution in combination with the pinhole diameter $(a)$ and $x$-ray source-size $(\Delta S)$. The simple expression for spectral resolution is given by combining the geometric resolution limited by blurring due to the finite source size and ideal diffractive resolution in quadrature (Sailaja 1998)

$$
\Delta \lambda=\sqrt{\frac{d}{n}\left(\frac{\Delta S+a}{R_{1}}+\frac{a}{R_{2}}\right)^{2}+\left(\frac{\lambda d}{n a}\right)^{2}}
$$

where $\mathrm{n}$ is the diffraction order. For typical inertial fusion experiments a source aperture is required to limit $\Delta S \leq 100 \mu \mathrm{m}$ in order to achieve a useful spectral resolution. Upon simple inspection of equation 2 it is clear that for wavelengths $\lambda=2-6 \mathrm{~nm}$, the resolution is always dominated by the geometric blurring due $\Delta S$, and the diffractive term contributes at most $5-10 \%$ for the lowest photon energies.

Sailaya et al. discuss the optimal resolution that can be obtained given a fixed detector distance $\left(R_{I}+R_{2}\right)$, illustrating that the spectral resolution improves with greater source-detector distance, becoming dominated by the diffractive term. While this optimum is important the $\Delta \lambda$ minimum is typically broad and must be considered in conjuction with the $\mathrm{x}$-ray signal typically decreasing as $1 /\left(R_{1}+R_{2}\right)^{2}$.

To optimise $R_{1}$, the spatial imaging requirements must also be considered. For a source apertured to $2.0 \mathrm{x}$ $0.1 \mathrm{~mm}$, the maximum magnification is limited by the $7.5 \mathrm{~mm}$ MCP strip height to $\sim 3$; this sets $\mathrm{R}_{1}=400 \mathrm{~mm}$. Given a reasonable pinhole imaging aperture of $a_{p h}=50 \mu \mathrm{m}$, the resulting spatial resolution would be $65 \mu \mathrm{m}$ or 30 resolution elements across the $2 \mathrm{~mm}$ aperture. However, using equation 2, this produces a spectral resolution of $\Delta E=20 \mathrm{eV}$ at $300 \mathrm{eV}$, which is a very limited number of resolution elements across the full spectral range, and too large to resolve typical absorption features expected in the laserplasmas. Increasing $R_{2}$ by a factor of two roughly doubles the spectral resolution, providing a good trade off with a spatial resolution of 24 resolution elements in $2 \mathrm{~mm}$.

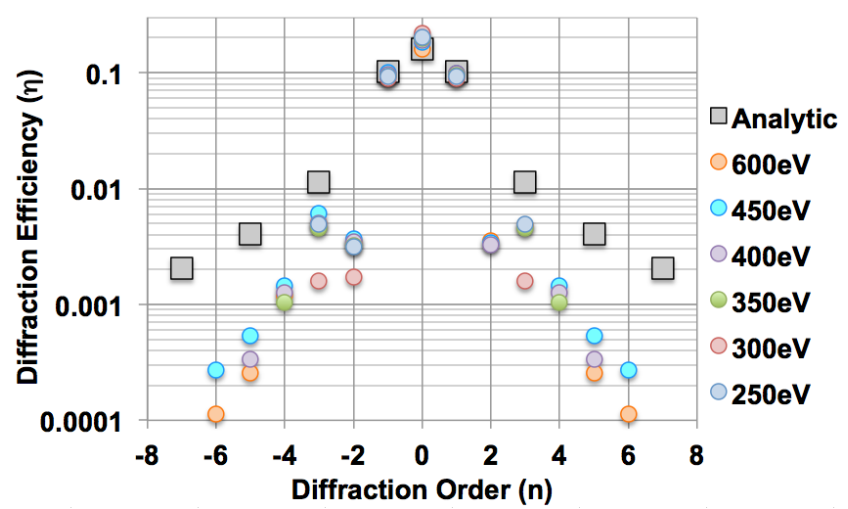

Figure 2 - Diffraction Efficiency measured between 250-600eV compared to the analytical expression assuming opaque grating bars.

\section{DIAGNOSTIC CHARACTERISATION}

To qualify the spectrometer performance a suite of measurements were made using the VUV beamline (U3C) at the NSLS (Bartlett 1988) and the Trident Laser Facility at LANL (Moncur 1995) and NIF (Moses 2009).

Due to the broad-band nature of a typical thermal x-ray emission spectrum, and the small-angle dispersion of the gratings, at any one spatial location on the detector, there will be a contribution from the $\mathrm{n}=1$ for $\lambda_{1}, \mathrm{n}=2$ from $\lambda_{1} / 2$, $\mathrm{n}=3$ from $\lambda_{1} / 3$ etc. Consequently it is necessary to characterise the contribution from higher-orders to the 
measured spectrum to be able to accurately infer the spectrum from the raw diffraction data. Using Fraunhofer diffraction theory it can be analytically shown that the diffraction efficiency for a transmission grating changes with diffracted order as (Eidmann 1990):

$$
\eta_{n}=\left[\frac{\sin \left(\frac{n \pi g}{d}\right)}{n \pi}\right]^{2} \ldots(3)
$$

where $\mathrm{g}$ is the gap between opaque bars. Since the nanometre size-scale of the grating structures typically have some degree of imperfections that would modify $\eta$, using beamline $\mathrm{U} 3 \mathrm{C}$ at the NSLS, we measure $\eta$ for photon energies between 250 and $600 \mathrm{eV}$. Figure 2 shows the results for one pinhole-grating. Data from 16 pinhole-grating assemblies was taken, obtaining good statistics on 'good' and 'bad' grating performance depending on grating defects and/or damage. The overall transmission of the grating is $40 \%$, with on average $10 \%$ being diffracted to $1^{\text {st }}$ order. $2^{\text {nd }}$ order contributions are suppressed by the even-function periodicity of the grating structure as can be seen from equation 3 when $2 g=d$. For all 16 pinhole-gratings the $3^{\text {rd }}$ order contributions are at most $0.7 \%$. As can be seen in Figure 2 this data compares favourably with the analytic expression with even high-orders being suppressed. Evidence of the odd orders is shown in the data, which is normalised to conserve energy. Error bars are smaller than the plotted points. By combining $\eta$, with the spectral dependence of the MCP quantum efficiency and thin $0.1 \mu \mathrm{m}$ Al filter, used as a light-block, then it can be shown that for a spectrally uniform source over the wavelength range above $300 \mathrm{eV}$, more than $90 \%$ of the measured signal originates from the $1^{\text {st }}$ order.

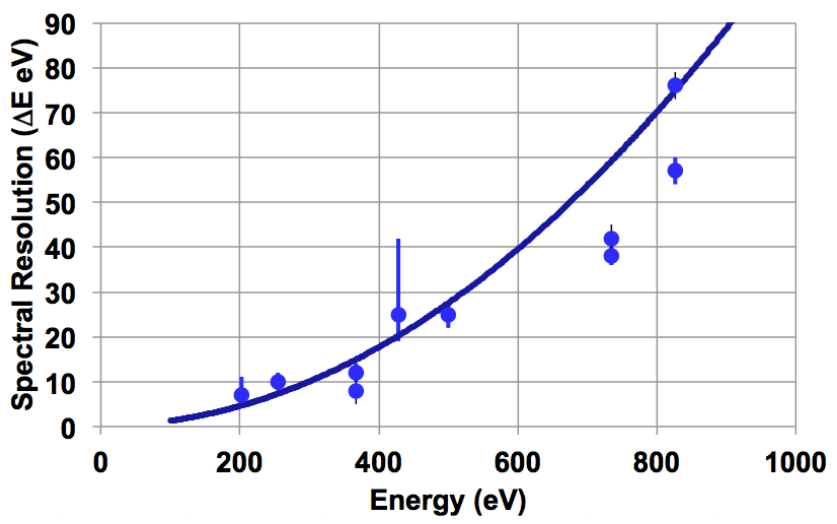

Figure 3 - Spectral Resolution measured using H-like and He-like emission lines. Analytical fit from equation 2 shown for $100 \mu \mathrm{m}$ source size.

Also critical to correctly interpreting the raw diffracted image is an accurate measurement of the spectral resolution; here defined as the FWHM of a Gaussian lineshape. Experiments were performed using the Trident Laser to irradiate various materials at $1 \times 10^{15} \mathrm{~W} / \mathrm{cm}^{2}$ using a $1.2 \mathrm{~ns}$, $527 \mathrm{~nm}$ laser pulse. Using elements from Boron -toFluorine this produced the characteristic $\mathrm{x}$-ray transitions from $n=2$, to $n=1$ ground state, $H$-like and He-like ions at photon energies between 200 and $830 \mathrm{eV}$. The measured line-width can be used to interrogate the spectral resolution of the diagnostic since the line-width of these transitions is much less than the diagnostic resolution. A 50 or $100 \mu \mathrm{m}$ pinhole was placed approx. $1 \mathrm{~mm}$ from the $\mathrm{x}$-ray source to limit the size of the emission region seen by the transmission grating spectrometer.

The results using a $100 \mu \mathrm{m}$ source-limiting pinhole are plotted in Figure 3 together with a line representing the result from equation 2 . The line-width is calculated by performing a $\chi^{2}$ Gaussian best-fit to the data for each emission line. Error bars represent the width corresponding to a Gaussian fit with $\chi^{2}+1$.

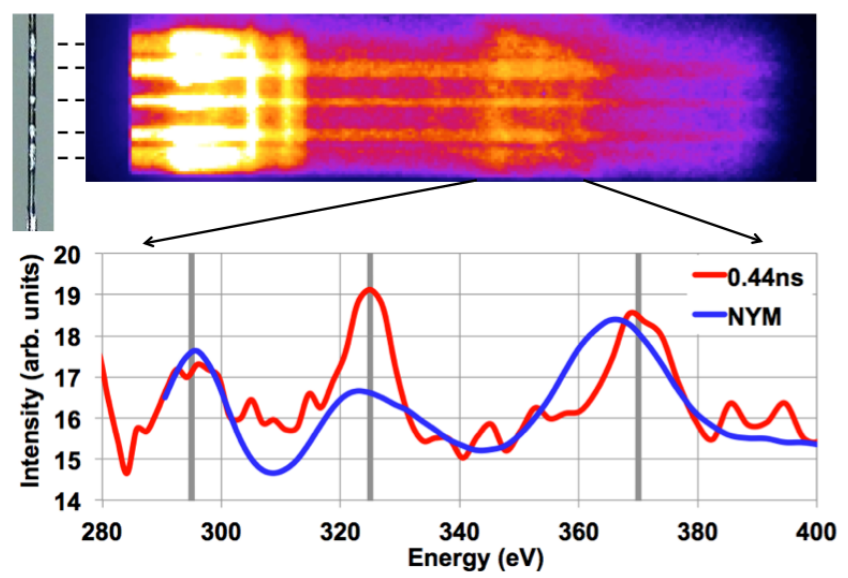

Figure $4-\mathrm{SiO}_{2}$ quartz fibre spectrum at $4 \times 10^{14} \mathrm{~W} / \mathrm{cm}^{2}$ (red). NYM simulations plotted (blue).

\section{DIAGNOSTIC PERFORMANCE}

To verify the operation of the spectrometer when coupled to the NIF GXD, a dedicated NIF shot (N120117003-999) irradiated a quartz fibre with $\mathrm{Cr}$ deposits at varying spatial locations along the fibre length, at two timeseparated intensities of $5 \times 10^{14}$ and $5 \times 10^{15} \mathrm{~W} / \mathrm{cm}^{2} . \Delta \mathrm{S}$ was limited by the $100 \mu \mathrm{m}$ fibre diameter. Figure 4 shows an image from the GXD. Plotted with the pre-shot simulations using the radiation hydrodynamics code NYM (Roberts 1980), the spectrum between $300-400 \mathrm{eV}$ shows much of the expected structure from Si 3-2 and 4-2 transitions and will be the discussed in more detail elsewhere (Guymer n.d.). Spatial line-outs of the image confirm that the FWHM spatial resolution is $<100 \mu \mathrm{m}$.

\section{CONCLUSIONS}

An optimised design for a transmission grating spectrometer for use on NIF to measure the soft x-ray spectrum between 200 and $700 \mathrm{eV}$ has been presented. Calibration results demonstrate the spectral resolution and diffraction efficiency perform better than expected compared to their analytic expectations. Qualification of the diagnostic on NIF shows the spectral and spatial performance is as predicted and that spectral features can be identified to $\pm 10 \%$.

\section{ACKNOWLEDGEMENTS}

We are grateful to D. Schmidt and the target preparation staff at LANL, support from the LANL Trident Laser facility, B. Dong at the NSLS, and shot operations staff at 
the NIF. This work was jointly supported by the Ministry of Defence, Los Alamos national laboratory and was performed under the auspices of the US. Department of Energy by Lawrence Livermore National Laboratory under contract DE-AC52-07NA273444. British Crown Copyright (2012)

\section{REFERENCES}

1. Back, C. A. "Diffusive, supersonic x-ray transpoer in radiatively heated foan cylinders." Physics of Plasmas 7, no. 5 (May 2000): 2126.

2. Bartlett, R. J., Trela, W. J., Michaud, F. D. "Characteristic and performance of the Los Alamos VUV beam line at the NSLS." Nuclear Instruments and Methods A 266 (1988): 199.

3. Ceglio, N.M., Kauffman, R.L., Hawryluk, A.M., Medecki, H. "Timeresolved x-ray transmission grating spectrometer for studying laserproduced plasmas.” Applied optics 22, no. 2 (1983): 318.

4. Eidmann, K., Kuhne, M., Muller, P., Tsakiris, G.D. "Characterisation of Pinhole Transmission Gratings.” Journal of X-ray Science and Technology 2 (1990): 259.

5. Guymer, T.,. "to be published."

6. Haynam, C A. "National Ignition Facility laser performance status." Applied Optics 46, no. 16 (June 2007): 3276.

7. Kline, J.L. "The first measurements of soft x-ray flux from ignition scale Hohlraums at the national Ignition Facility using DANTE." Review of Scientific Instruments 81 (2010): 10E321.

8. Moncur, N.K., Johnson, R.P., Watt, R.G., Gibson, R.B. "Trident: a versatile high-power $\mathrm{Nd}$ : glass laser facility for inertial confinement fusion experiments." Applied Optics 35 (1995): 4274.

9. Roberts, P.D., Rose, S. J., Thompson, P. C., Wright, R. J. "The stability of multiple-shell ICF targets." Journal of Physics D 13 (1980): 1957.

10. Rochau, G.A., Bailey, J. E.,. "Energy dependent sensitivity of microchannel plate detectors." Rev. Sci. Inst. 77 (2006): 10E3223.

11. Sailaja, S., Arora, V., Kumbhare, S. R., Gupta, P.D., Fedin, D. A., Rupasov, A. A., Shikanov, A. S. "A simple XUV transmission grating spectrograph with sub-angstrom resolution for laser-plasma interaction studies.” Measurement Science Technology 9 (1998): 1462.

12. Ze. Review od Scientific Instruments 63 (1992): 5124. 